\title{
Ionization-Induced Electron Trapping in Ultrarelativistic Plasma Wakes
}

\author{
E. Oz, ${ }^{1}$ S. Deng, ${ }^{1}$ T. Katsouleas, ${ }^{1}$ P. Muggli, ${ }^{1}$ C. D. Barnes, ${ }^{2}$ I. Blumenfeld, ${ }^{2}$ F. J. Decker, ${ }^{2}$ P. Emma, ${ }^{2}$ M. J. Hogan, ${ }^{2}$ \\ R. Ischebeck, ${ }^{2}$ R. H. Iverson, ${ }^{2}$ N. Kirby, ${ }^{2}$ P. Krejcik, ${ }^{2}$ C. O'Connell, ${ }^{2}$ R. H. Siemann, ${ }^{2}$ D. Walz, ${ }^{2}$ D. Auerbach, ${ }^{3}$ \\ C. E. Clayton, ${ }^{3}$ C. Huang, ${ }^{3}$ D. K. Johnson, ${ }^{3}$ C. Joshi, ${ }^{3}$ W. Lu, ${ }^{3}$ K. A. Marsh, ${ }^{3}$ W. B. Mori, ${ }^{3}$ and M. Zhou ${ }^{3}$ \\ ${ }^{1}$ Department of Electrophysics and Electrical Engineering, USC, Los Angeles, California 90089, USA \\ ${ }^{2}$ Stanford Linear Accelerator Center, SLAC, Menlo Park, California 94025, USA \\ ${ }^{3}$ Department of Electrical Engineering, UCLA, Los Angeles, California 90095, USA \\ (Received 23 October 2006; published 23 February 2007)
}

\begin{abstract}
The onset of trapping of electrons born inside a highly relativistic, 3D beam-driven plasma wake is investigated. Trapping occurs in the transition regions of a $\mathrm{Li}$ plasma confined by $\mathrm{He}$ gas. Li plasma electrons support the wake, and higher ionization potential He atoms are ionized as the beam is focused by $\mathrm{Li}$ ions and can be trapped. As the wake amplitude is increased, the onset of trapping is observed. Some electrons gain up to $7.6 \mathrm{GeV}$ in a $30.5 \mathrm{~cm}$ plasma. The experimentally inferred trapping threshold is at a wake amplitude of $36 \mathrm{GV} / \mathrm{m}$, in good agreement with an analytical model and PIC simulations.
\end{abstract}

DOI: 10.1103/PhysRevLett.98.084801

The self-trapping of electrons in plasma waves is a topic of much contemporary interest. In their seminal paper Akhiezer and Polovin [1] showed that, for one dimensional relativistic plasma waves in a cold plasma with density $n_{p}$, fluid theory breaks down when the wave amplitude reaches

$$
E_{\max }=\sqrt{2\left(\gamma_{\phi}-1\right)} m c \omega_{p} / e,
$$

where $\omega_{p}=\sqrt{\frac{n_{p} e^{2}}{m \epsilon_{0}}}$ is the plasma frequency, $\gamma_{\phi}=$ $\left(1-v_{\phi}^{2} / c^{2}\right)^{-1 / 2}$, and $v_{\phi}$ is the wave phase velocity. A physical interpretation of this limit for a nonrelativistic plasma wave was given by Dawson [2] who associated it with wave breaking and self-trapping of plasma electrons due to self steepening of the wave. Calculation of $E_{\max }$ in a 1D, warm plasma leads to a small correction to the cold plasma results [3-6]. Self-trapped, high energy electrons have been routinely observed in laser-driven plasma waves with moderate $\gamma_{\phi}$ [7-10]; however, no systematic study of the onset of the trapping process has been carried out.

In this Letter we explore a new trapping mechanism: the onset of trapping of electrons that are ionized inside a highly relativistic plasma wave (wake). The wake is driven by the passage of a short electron bunch through a lithium (Li) vapor column confined by a helium $(\mathrm{He})$ gas. The wake field amplitude is controlled by varying the peak current of the drive electron bunch. We show that in the experiment, there is a well-defined threshold for the onset of electron trapping in an ionizing plasma, at a value much lower than that given by Eq. (1). A trapping threshold for these newly ionized particles is derived and is in excellent agreement with that obtained in numerical simulations and with that inferred from experimental measurements. Simulations also indicate that the trapped particles are He electrons born near the beam axis inside the wake itself.
PACS numbers: 41.75.Ht, 41.75.Lx, 52.25.Jm, 52.40.Mj

The experiment uses the ultrarelativistic $(28.5 \mathrm{GeV})$ ultrashort $(<100 \mathrm{fs})$ electron bunches with approximately $1.6 \times 10^{10}$ electrons available at the Stanford Linear Accelerator Center. A schematic of the experiment is shown in Fig. 1(a). The electron beam is focused near the entrance of a Li vapor column of density $n_{0}$ contained in a heat-pipe oven [11]. The $\mathrm{Li}$ is confined to the oven hot region by a He buffer gas which is at room temperature away from the hot region. The total pressure is constant along the oven. The density profile of the $\mathrm{Li}$ vapor measured along the oven, as well as the inferred He density are

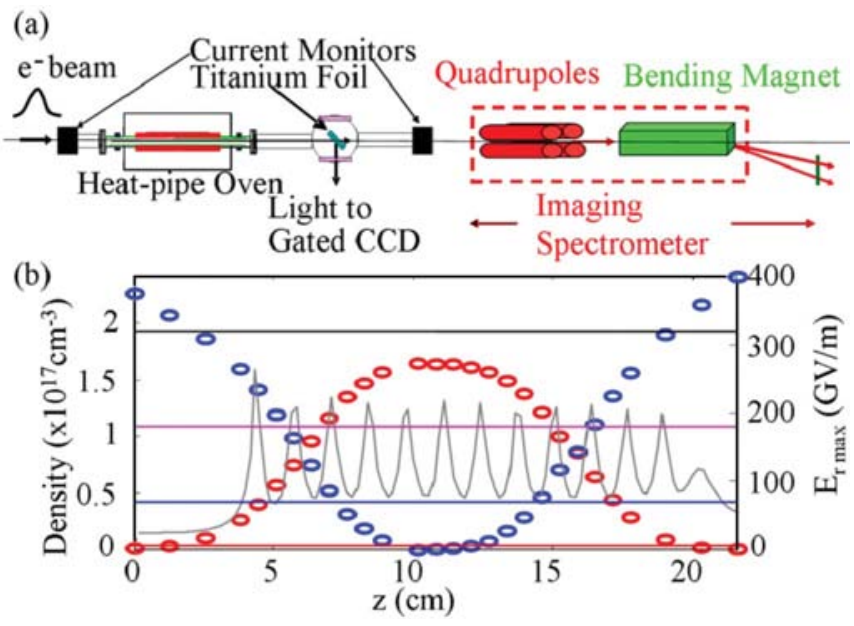

FIG. 1 (color). (a) Schematic of the experimental setup. (b) Measured longitudinal density of Li vapor (red circles) and inferred He gas density (blue circles) in the heat-pipe oven for $n_{0}=1.6 \times 10^{17} \mathrm{~cm}^{-3}$. The gray curve is the calculated maximum radial field of the bunch as it is focused and propagates along the plasma. The multiple peaks are due to the periodic oscillations of the beam's transverse envelope. The red, blue, magenta, and black lines are the field ionization thresholds for $\mathrm{Li}, \mathrm{He}, \mathrm{He}^{+}$, and $\mathrm{Li}^{+}$, respectively. 
shown in Fig. 1(b) for the case $n_{0}=1.6 \times 10^{17} \mathrm{~cm}^{-3}$. The plasma length is $\approx 10 \mathrm{~cm}(\mathrm{FWHM})$. The plasma is created through tunnel ionization [12] of the low ionization potential $(5.39 \mathrm{eV}$, first electron) $\mathrm{Li}$ vapor by the large radial space charge field of the drive bunch where the field exceeds the threshold value of $E_{\mathrm{Li}}=5 \mathrm{GV} / \mathrm{m}$ [13]. The plasma density, $n_{p}$ is therefore equal to the local neutral $\mathrm{Li}$ density [Fig. 1(b)]. The electron bunch charge is measured before and after the plasma using current transformers. The prompt visible radiation emitted by the electrons is extracted from the beam line using a $1 \mu \mathrm{m}$ thick Ti foil at $45^{\circ}$, and is recorded using a gated charge coupled device (CCD) camera. It is a combination of bremsstrahlung radiation emitted by the particles along the plasma, Cherenkov radiation emitted along the plasma and buffer gas, and transition radiation emitted at the extraction foil. After the plasma, a magnetic spectrometer images and disperses the drive beam to yield single-bunch energy spectra from which we can infer wakefield amplitudes with a resolution of the order of $1 \%$ of the incoming beam energy [14].

The experiment is carried out in the nonlinear or blowout regime of the plasma wakefield accelerator, in which the focused beam density exceeds the plasma density. As the drive electron bunch enters the Li vapor, its space charge field first ionizes $\mathrm{Li}$ and then expels all the plasma electrons out of the bunch volume. The positive ion charge left behind the bunch head strongly focuses the electron beam. In expelling the plasma electrons, the head and core bunch particles lose energy to the plasma wake. The plasma electrons rush back to the beam axis, approximately one plasma period later. This creates an on-axis negative charge density spike that can accelerate the electrons in the back of the bunch and also trap plasma electrons. A peak energy gain of about $4 \mathrm{GeV}$, the largest published to date in any plasma accelerator, has been observed in a $10 \mathrm{~cm}$ long, $2.8 \times 10^{17} \mathrm{~cm}^{-3}$ plasma [14], corresponding to an accelerating gradient of $\approx 40 \mathrm{GV} / \mathrm{m}$. As the beam is focused by the plasma ion column to a size smaller than that at the plasma entrance, its space charge field increases as shown in Fig. 1(b) and can become large enough to ionize the first electron of the He buffer gas (24.6 eV ionization potential). This occurs in the $\mathrm{He}$ to $\mathrm{Li}$ transition regions $[0<z<9 \mathrm{~cm}$ and $12<z<20 \mathrm{~cm}$ in Fig. 1(b)] of the oven where the bunch field exceeds the threshold value $E_{\mathrm{He}}=70 \mathrm{GV} / \mathrm{m}$. These newly ionized He electrons are born inside the plasma wake that is supported by the $\mathrm{Li}$ electrons, and we show later that they can therefore be trapped and accelerated. The trapped plasma electrons exit the plasma with the drive bunch and are detected in the form of excess charge and light.

Experimentally, the onset of trapping is observed by varying the bunch length or peak current with a constant number of particles. At a given plasma density the wake amplitude is inversely related to the bunch length [15]. There is a threshold in wakefield amplitude (or beam energy loss) at which we observe a sudden increase in the amount of charge detected after the plasma $\left[W_{\text {loss }} \approx\right.$ $0.9 \mathrm{GeV}$ in Fig. 2(a)]. The wake field amplitude is related to the average energy loss ( $\left.W_{\text {loss }}\right)$ by the drive beam electrons. Appearance of this excess charge also coincides with the abrupt increase in the amount of light collected downstream of the plasma [Fig. 2(b)]. The increase in total charge in Fig. 2(a) is on the order of that in the drive beam. The relative amount of light increase is larger than the relative charge increase and is the result of the coherent emission of light by short temporal structures of the trapped particles.

To gain insight into the physics of trapping, we perform 2D cylindrically symmetric particle-in-cell (PIC) simulations using the numerical code OSIRIS [16] for the full parameters of the experiment, including the $\mathrm{Li}$ and $\mathrm{He}$ profiles of Fig. 1(b), as well as the field ionization process [13]. Figure 3(a) shows that the Li electrons support the wake. The He electrons are trapped and bunched in a short $(\approx 2 \mu \mathrm{m}$ long) region near the peak accelerating field of the first wake bucket $[\xi \approx 230 \mu \mathrm{m}$ in Fig. 3(b)]. The OSIRIS simulation uses a window moving at the speed of light $c$ therefore $\xi=z-c t$; however, we derive the analytical threshold expression for $\xi=z-v_{\phi} t$, and therefore, while using simulation results we are approximating $c$ as $v_{\phi}$, note that for a $28.5 \mathrm{GeV}$ beam $v_{\phi} \approx c$. The longitudinal momentum of the $\mathrm{Li}$ electrons oscillating in the wake [Fig. 3(c)] is much less than that of the He electrons [Fig. 3(d)]. The He electrons are focused near the beam axis and in this case reach energies up to $2.5 \mathrm{GeV}$ after the $10 \mathrm{~cm}$ long plasma. Trapped particle energies up to $\approx 2.5 \mathrm{GeV}$ after a $10 \mathrm{~cm}$ long, and $\approx 7.6 \mathrm{GeV}$ after a $30.5 \mathrm{~cm}$ long, $2.7 \times 10^{17} \mathrm{~cm}^{-3}$ plasma have been measured in the experiment.

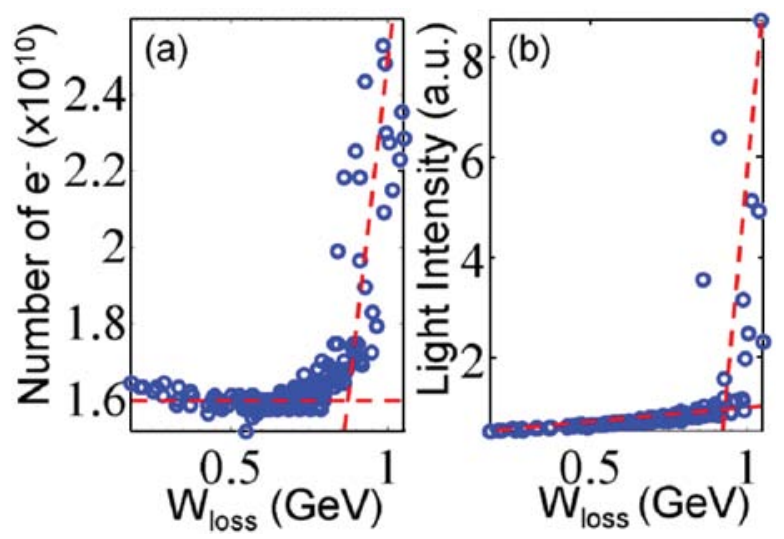

FIG. 2 (color). (a) Number of electrons measured after the plasma and (b) relative amount of visible continuum light emitted by these particles versus the average beam energy loss. Note, the charge fluctuations below threshold on (a) are also present in the incoming charge as measured before the plasma. The plasma density is $1.6 \times 10^{17} \mathrm{~cm}^{-3}$ [corresponding to the plasma density profile of Fig. 1(b)]. 


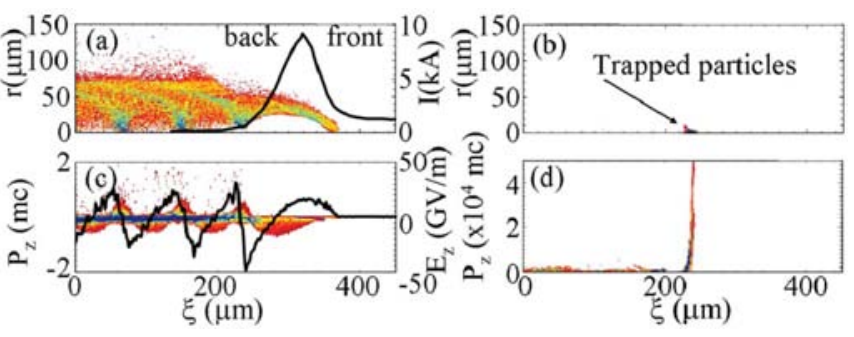

FIG. 3 (color). 2D OSIRIS simulation results using the Li vapor and $\mathrm{He}$ gas profiles and density of Fig. 1(b). The left panels correspond to Li and the right to He electrons. Figures (a) and (b) are real space densities $(r-z)$ at $z=11.3 \mathrm{~cm}$ [Fig. 1(b)] and (c) and (d) are the corresponding phase space densities $\left(p_{z}-z\right)$ at $z=$ $11.3 \mathrm{~cm}$ and $z=22 \mathrm{~cm}$, respectively. The line plot in (c) is the on-axis wakefield $E_{z}$. The beam [black line in (a)] has $1.88 \times$ $10^{10}$ electrons, a Gaussian transverse profile with $\sigma_{r}=10 \mu \mathrm{m}$, and a longitudinal profile with FWHM $\approx 65 \mu \mathrm{m}$. The simulation was performed on a moving $500 \times 600$ grid $(\Delta z \times \Delta r$, $1 \mu \mathrm{m} \times 0.5 \mu \mathrm{m})$ with 25 beam particles/cell and 1 of each gas atoms/cell. Simulations with higher spatial resolution and more particles/cell gave similar results.

Density gradients can also cause particle trapping [17]; however, this trapping only occurs in the case of very sharp decreasing plasma density gradients where the density scale length is smaller than the plasma collisionless skin depth $c / \omega_{p}$. This condition is not satisfied in our experiment [Fig. 1(b)].

Having established in simulations that the trapped particles are He electrons ionized inside the wake, we inves-

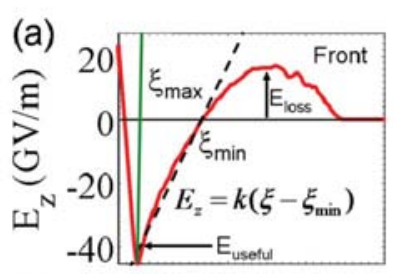

(b)
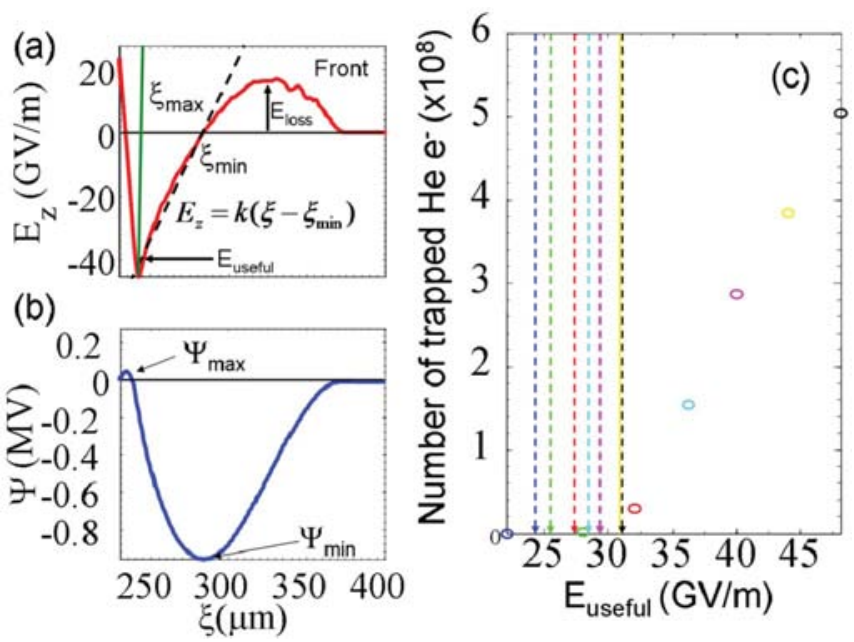

FIG. 4 (color). (a) On-axis wakefield (continuous red line), $E_{z}$, the same as in Fig. 3(c) and fit to the linear part of $E_{z}$ (black dashed line) between $\xi_{\min }$ and $\xi_{\max }$. (b) Wake potential $\Psi$. (c) Number of trapped He electrons (circles) as a function of the peak accelerating field from short simulations with $n_{0}=$ $1.6 \times 10^{17} \mathrm{~cm}^{-3}$, and a number of electrons increasing from 0.4 to 1 times $1.8 \times 10^{10} e^{-}$. The dashed vertical lines show the trapping threshold calculated from Eq. (3). Circles and lines of the same color correspond to the same simulation. The same current profile as in Fig. 3 has been used but $\sigma_{r}=2.4 \mu \mathrm{m}$. tigate the threshold for the onset of trapping in a 3D highly relativistic wake in the presence of an ionizing gas. We begin with the constant of motion for particles in arbitrary wave potentials of the form, $\mathbf{A}=\mathbf{A}\left(x, y, z-v_{\phi} t\right), \Phi=$ $\Phi\left(x, y, z-v_{\phi} t\right)$. This constant is obtained by subtracting the longitudinal equation of motion from the rate of energy gain equation for the plasma particles [18]. It takes the form $\gamma m c-\frac{v_{\phi}}{c} P_{z}+q \frac{\Psi}{c}=$ constant where $\gamma$ is the particle's Lorentz factor and $P_{z}$ is the longitudinal momentum of the trapped particle of charge $q$. The potential function $\Psi=\Phi-v_{\phi} A_{z}$ is related to the longitudinal wakefield by $E_{z}=-\partial_{\xi} \Psi$ where $\xi=z-v_{\phi} t$. Evaluating the constant for particles ionized at rest $\left(P_{z}=0, \gamma=1\right)$, near the axis, and at a phase such that $\Psi=\Psi_{i}$ [Figs. 4(a) and 4(b)] gives the constant to be $m c+q \frac{\Psi_{i}}{c}$. The trapping condition follows from the requirement that the electron velocity $v_{z}$ reaches the wake phase velocity $v_{\phi}$ by the time the electron slips back to the peak of the potential at $\Psi=\Psi_{\max }$. Therefore, we have $m c-\gamma m c+\gamma m v_{\phi}^{2} / c \leq \frac{q}{c} \times$ $\left(\Psi_{\max }-\Psi_{i}\right)$. Particles born near the axis have a perpendicular velocity that remains much smaller than their parallel velocity, and the trapping condition is then

$$
m c^{2}\left(1-\frac{1}{\gamma_{\phi}}\right) \leq q\left(\Psi_{\max }-\Psi_{i}\right) .
$$

Therefore the lowest threshold for trapping occurs for particles born where $\Psi=\Psi_{\min }$. A theory for wakes in nonlinear blow out regime shows that $E_{z}$ is nearly linear over a large region of $\xi$ from $\xi_{\text {min }}$ to $\xi_{\max }$ [Fig. 4(a)] [19]. We thus write $\tilde{E}_{z} \approx k\left(\tilde{\xi}-\tilde{\xi}_{\text {min }}\right)$ where we obtain $k$ from linear fitting to the $E_{z}$ field obtained from PIC simulations; tildes indicate dimensionless quantities $[\tilde{E}=$ $\left.E /\left(m c \omega_{p} / e\right), \tilde{\xi}=\xi /\left(c / \omega_{p}\right)\right]$. Integrating the linear $E_{z}$ field from $\xi_{\min }$ to $\xi_{\max }$ (Fig. 4) gives the rhs of Eq. (2). Therefore the trapping threshold is (for $\gamma_{\phi} \gg 1$ )

$$
\left|\tilde{E}_{\max }\right| \geq \sqrt{2 k}
$$

Simulations are used to calculate $k$ and $\tilde{E}_{\text {max }}$. The wake amplitude is controlled by varying the drive bunch charge. To reduce the simulation time while investigating the threshold, a short plasma profile is used where the plasma density is quickly ramped up to match the peak density of the measured profile seen in Fig. 1(b), and an arbitrarily small amount of $\mathrm{He}$ is introduced along the Li profile. The beam and trapped electrons exit the plasma through a short vacuum section. In Fig. 4(c) we plot the number of trapped $\mathrm{He}$ electrons as a function of the accelerating field. The useful accelerating field $E_{\text {useful }}$ is defined as the electric field amplitude at $\xi_{\max }$ extrapolated from the linear fit to the electric field starting at $\xi_{\min }$, where $E_{z}=0$. This definition of the accelerating field also gives the value of $k$ [Fig. 4(a)]. Trapping is observed when the $E_{\text {uselful }}>$ $30 \mathrm{GV} / \mathrm{m}$. Both in these simulations and in the experiment [Fig. 2(a)] there is a sudden increase in the number of trapped particles when the wake amplitude is increased. To 
relate the peak electric field to the energy loss we find the average energy loss in the simulation of Fig. 3 which is carried out for the exact parameters of the experiment. The peak field of $40 \mathrm{GV} / \mathrm{m}$ in that simulation corresponds to an average energy loss of $1 \mathrm{GeV}$. Using this proportionality factor, the observed trapping threshold at $E_{\text {loss }} \approx 0.9 \mathrm{GeV}$ in Fig. 2(a) implies a trapping threshold field of $\approx 36 \mathrm{GV} / \mathrm{m}$. Note, simulations without He show that no $\mathrm{Li}$ electrons are trapped over the range of longitudinal wakefields of Fig. 4(c). Note also that the number of trapped electrons in Fig. 4 is much smaller than that in Fig. 2(a). It is the result of trapping of low density He over the short plasma length used in the simulation while in the experiment electrons are trapped in both buffer regions of the oven (Fig. 1).

Equation (3) is also plotted in Fig. 4(c) (dashed vertical lines) using the values of $k$ obtained from $E_{z}$ of the short simulations described above. Trapping is observed only for simulations where the peak accelerating field is higher than the trapping threshold [Eq. (3)], i.e., when the colored circles are to the right of the lines of same color in Fig. 4. The onset of trapping occurs at $E_{\text {useful }} \approx 30 \mathrm{GV} / \mathrm{m}$. The value of $k$ approaches $\frac{1}{2}$ in the limit of extreme blowout [20]. Thus, the trapping threshold for electrons ionized inside the ultrarelativistic wave becomes $E_{\max } \approx$ $m c \omega_{p} / e$, independent of $\gamma_{\phi}$ and surprisingly the same as that in a cold, 1D, nonrelativistic plasma [2]. In the present experiment where $\gamma_{\phi} \approx 56000$ this value is a factor $\sqrt{2\left(\gamma_{\phi}-1\right)} \approx 335$ times smaller than that given by Eq. (1), i.e., for a 1D wake in a preionized plasma. Note, without field ionization inside the wake, all electrons enter the wake from $\Psi=0[\xi>370 \mu \mathrm{m}$ on Figs. 4(a) and 4(b)] rather than the lower value near $\Psi_{\min }$. Therefore, the trapping threshold is expected to be significantly larger to trap electrons in a preionized plasma or the field ionized $\mathrm{Li}$ electrons in this case. However, no analytic expression is readily obtained for these cases since it involves integrating the field $E_{z}$ over the nonlinear region in front of the zero crossing in Fig. 4(a). Also note that the trapping threshold is insensitive to the asymmetries in the beam since the ionized volume and the wake amplitude are insensitive to beam asymmetries as long as the beam radius is much smaller than $c / w_{p}$.

In summary, physics of particle trapping in 3D relativistic plasma waves in the presence of field ionization of the ambient gas or vapor has been examined. A new analytical model was developed that shows that the onset of trapping for particles born inside the wake is expected at a wake amplitude of $30 \mathrm{GV} / \mathrm{m}$ for $n_{p}=1.6 \times 10^{17} \mathrm{~cm}^{-3}$. Numerical simulations (2D cylindrically symmetric) with the parameters of the experiment predict a trapping threshold at $30 \mathrm{GV} / \mathrm{m}$. Simulations also show that the trapping threshold is insensitive to the incoming beam energy as expected from Eq. (2) (for $\gamma_{\phi} \gg 1$ ). They identify the trapped particles as He electrons born inside the ultrarelativistic wake sustained by the Li electrons. Experimentally we observed this threshold at a wake amplitude of $36 \mathrm{GV} / \mathrm{m}$ as deduced from the observed beam energy loss. These three trapping threshold values are in excellent agreement with each other. Experimental measurements show that the trapped particles gain up to $7.6 \mathrm{GeV}$ over a $30.5 \mathrm{~cm}$ long plasma. The observed energy distributions were typically very broad, but often with clear high energy peak or peaks. These results will be reported elsewhere. Understanding and control of this new trapping mechanism could lead to the production of ultrashort and ultrabright monoenergetic electron bunches with possible applications to advanced accelerators and light sources.

This work was supported by Department of Energy Contracts No. DE-AC02-76SF00515, No. DE-FG0392ER40745, No. DE-FG03-98DP00211, No. DE-FG0392ER40727, No. DE-AC-0376SF0098, and National Science Foundation Grants No. ECS-9632735, No. DMS9722121, and No. PHY-0078715, simulations were done at the USC Center for High Performance Computing and Communications (HPCC) and National Energy Research Scientific Computing Center.

[1] A. I. Akhiezer and R. P. Polovin, Sov. Phys. JETP 3, 696 (1956).

[2] J. M. Dawson, Phys. Rev. 113, 383 (1959).

[3] T. P. Coey, Phys. Fluids 14, 1402 (1971).

[4] W. B. Mori and T.C. Katsouleas, Phys. Scr. T30, 127 (1990).

[5] T. C. Katsouleas and W. B. Mori, Phys. Rev. Lett. 61, 90 (1988).

[6] J. B. Rosenzweig, Phys. Rev. A 38, 3634 (1988).

[7] A. Modena et al., Nature (London) 377, 606 (1995).

[8] D. Umstadter, J. K. Kim, and E. Dodd, Phys. Rev. Lett. 76, 2073 (1996).

[9] W. P.Leemans et al., IEEE Trans. Plasma Sci. 24, 331 (1996).

[10] C. Joshi and T. Katsouleas, Phys. Today 56, No. 6, 47 (2003).

[11] P. Muggli et al., IEEE Trans. Plasma Sci. 27, 791 (1999).

[12] C. L. O'Connell et al., Phys. Rev. ST Accel. Beams 9, 101301 (2006).

[13] M. V. Ammosov et al., Sov. Phys. JETP 64, 1191 (1986).

[14] M. J. Hogan et al., Phys. Rev. Lett. 95, 054802 (2005).

[15] W. Lu et al., Phys. Plasmas 12, 063101 (2005).

[16] R. Hemker, in Proceedings of the Fifth IEEE Particle Accelerator Conference (IEEE, Piscataway, NJ, 1999), p. 3672.

[17] H. Suk et al., Phys. Rev. Lett. 86, 1011 (2001).

[18] P. Mora and J. T. M. Antonsen, Phys. Plasmas 4, 217 (1997); the equation differs slightly because we transform to $z-v_{\phi} t$.

[19] W. Lu et al., Phys. Rev. Lett. 96, 165002 (2006).

[20] W. Lu et al., Phys. Plasmas 13, 056709 (2006). 